PROCEEDINGS OF THE AMERICAN MATHEMATICAL SOCIETY

Volume 124, Number 2, February 1996

\title{
ON RESULTS OF CZUBIAK-GUNDERSEN AND OSGOOD-YANG
}

\author{
HONG-XUN YI
}

(Communicated by Albert Baernstein II)

\begin{abstract}
This paper studies the problem of uniqueness of entire functions that share real zeros and real ones. An example is provided to show that a result of Czubiak and Gundersen is not completely correct. Results in this paper correct the result of Czubiak and Gundersen, and also correct a result of Osgood and Yang.
\end{abstract}

\section{INTRODUCTION AND MAIN RESULTS}

We say that two entire functions $f(z)$ and $g(z)$ share the value $c$ provided that $f(z)=c$ if and only if $g(z)=c$. We distinguish between sharing a value CM (counting multiplicities) and IM (ignoring multiplicities).

Let $\mathcal{K}$ be the class of all nonconstant entire functions which have only real zeros and real ones. T. P. Czubiak and G. G. Gundersen obtained the following result:

Theorem A $([1$, Theorem $])$. If $f$ and $g$ are in $\mathcal{K}$ and share 0 and 1 IM, then we necessarily have one of the following six cases where $a \neq 0$ and $b$ are real constants:

$$
f(z)=\frac{\sin ^{2}(p(a z+b))}{\sin ^{2}(a z+b)} \quad \text { and } \quad g(z)=\frac{\sin (p(a z+b))}{\sin (a z+b)} e^{i(p-1)(a z+b)}
$$

for $p=-2$ and for $p=-3$;

$$
f(z)=1-\frac{(g(z)-1)^{2}}{e^{2 p i(a z+b)}} \quad \text { and } \quad g(z)=\frac{\sin (p(a z+b))}{\sin (a z+b)} e^{i(p-1)(a z+b)}
$$

for $p=3$ and for $p=4$.

From Theorem A Czubiak and Gundersen obtained the following result.

Theorem B ([1, Corollary 1$])$. If $f$ and $g$ are in $\mathcal{K}$ and share 0 and $1 C M$, then $f \equiv g$.

Received by the editors May 25, 1994 and, in revised form, September 13, 1994.

1991 Mathematics Subject Classification. Primary 30D35, 30D20.

Key words and phrases. Entire function, uniqueness theorem, shared value, real zeros.

This research was partially supported by the National Natural Science Foundation of China.

(C)1996 American Mathematical Society 
The above results of Czubiak and Gundersen, however, are not true for $f(z)=$ $\left(\frac{1}{2}+\mathrm{id}\right)\left(1-e^{i z}\right)$ and $g(z)=\left(\frac{1}{2}-\mathrm{id}\right)\left(1-e^{-i z}\right)$, where $d$ is a real constant.

In this note, we prove the following theorems which are corrections of Theorem A and Theorem B.

Theorem 1. If $f$ and $g$ are in $\mathcal{K}$ and share 0 and 1 IM, then $f$ and $g$ must satisfy exactly one of the following relations:

$$
f \equiv g
$$

$$
\begin{aligned}
& f(z)=\frac{\sin (a z+b)}{\sin (b-c)} e^{i(a z+c)}=\left(\frac{1}{2}+\mathrm{id}\right)\left(1-e^{2 i(a z+b)}\right), \\
& g(z)=\frac{\sin (a z+b)}{\sin (b-c)} e^{-i(a z+c)}=\left(\frac{1}{2}-\mathrm{id}\right)\left(1-e^{-2 i(a z+b)}\right),
\end{aligned}
$$

where $a, b, c, d$ are real constants, and $a \neq 0, \sin (b-c) \neq 0, d=\frac{1}{2} \cot (b-c)$;

$$
\begin{aligned}
& f(z)=\frac{\sin (p(a z+b))}{\sin (a z+b)} e^{i(p-1)(a z+b)}, \\
& g(z)=\frac{\sin (p(a z+b))}{\sin (a z+b)} e^{-i(p-1)(a z+b)},
\end{aligned}
$$

where $p$ is an integer, $a, b$ are real constants, and $p \neq 0,1, a \neq 0$;

$$
f(z)=\sin ^{2}(a z+b) \quad \text { and } \quad g(z)=-i \sin (a z+b) e^{i(a z+b)},
$$

where $a \neq 0$ and $b$ are real constants;

$$
f(z)=\frac{\sin ^{2}(p(a z+b))}{\sin ^{2}(a z+b)} \quad \text { and } \quad g(z)=\frac{\sin (p(a z+b))}{\sin (a z+b)} e^{i(p-1)(a z+b)},
$$

for $p=-2$ and for $p=-3$, where $a \neq 0, b$ are real constants;

$$
\begin{aligned}
& f(z)=1-\frac{\sin ^{2}((p-1)(a z+b))}{\sin ^{2}(a z+b)}, \\
& g(z)=\frac{\sin (p(a z+b))}{\sin (a z+b)} e^{i(p-1)(a z+b)},
\end{aligned}
$$

for $p=3$ and for $p=4$, where $a \neq 0, b$ are real constants.

Theorem 2. If $f$ and $g$ are in $\mathcal{K}$ and share 0 and $1 C M$, then $f$ and $g$ must satisfy exactly one of the relations (i), (ii), and (iii) of Theorem 1.

\section{SOME LEMMAS}

In order to prove our theorems, we need the following lemmas.

Lemma 1 (cf. [2, Corollary of Theorem 2]). If $f$ is in $\mathcal{K}$, then the order of $f$ is at most one.

Lemma 2 (cf. [2, Proposition 1]). If $f$ is in $\mathcal{K}$, and $f$ is not real for some real value of $z$, then $f$ is necessarily of one of the following two forms:

$$
f(z)=\frac{\sin (a z+b)}{\sin (b-c)} e^{i(a z+c)}, \quad \sin (b-c) \neq 0,
$$


where $a \neq 0, b$ and $c$ are real constants;

$$
f(z)=\frac{\sin (p(a z+b))}{\sin (a z+b)} e^{i(p-1)(a z+b)},
$$

where $p \neq 0,1$ is an integer, and $a \neq 0, b$ are real constants.

Lemma 3 ([3, Theorem 2]). Let $Q_{1}(z)$ and $Q_{2}(z)$ be nonconstant polynomials of the same degree. If

$$
f(z)=\frac{e^{Q_{1}(z)}-1}{e^{Q_{2}(z)}-1}
$$

is a nonconstant entire function, then

$$
Q_{1}(z)=p Q_{2}(z)+2 q \pi i
$$

where $p \neq 0,1$ and $q$ are integers.

\section{Proof of Theorem 2}

Since $f$ and $g$ are in $\mathcal{K}$, by Lemma 1 we have order $(f) \leq 1$ and $\operatorname{order}(g) \leq 1$. From the fact that $f$ and $g$ share the values $0,1 \mathrm{CM}$, we obtain

$$
\frac{f(z)}{g(z)}=e^{a_{1} z+b_{1}}, \quad \frac{f(z)-1}{g(z)-1}=e^{a_{2} z+b_{2}},
$$

where $a_{1}, a_{2}, b_{1}$, and $b_{2}$ are constants. Suppose that $f \not \equiv g$. Then from (1) we deduce that

$$
f(z)=\frac{e^{a_{2} z+b_{2}}-1}{e^{a_{3} z+b_{3}}-1}, \quad g(z)=\frac{e^{-\left(a_{2} z+b_{2}\right)}-1}{e^{-\left(a_{3} z+b_{3}\right)}-1},
$$

where $a_{3}=a_{2}-a_{1}, b_{3}=b_{2}-b_{1}$ are constants, and $e^{a_{3} z+b_{3}} \not \equiv 1, a_{2} \neq 0$.

Assume that $a_{3}=0$ and $e^{b_{3}}=K$. Then $K \neq 0,1$. From (2) we have

$$
f(z)=\frac{1}{K-1}\left(e^{a_{2} z+b_{2}}-1\right), \quad g(z)=\frac{K}{1-K}\left(e^{-\left(a_{2} z+b_{2}\right)}-1\right) .
$$

Hence, $z_{1}=\left(2 \pi i / a_{2}\right)-\left(b_{2} / a_{2}\right)$ and $z_{2}=z_{1}+\left(2 \pi i / a_{2}\right)$ are two zeros of $f$. Since $z_{1}$ and $z_{2}$ are real, we obtain $\operatorname{Re}\left(a_{2}\right)=\operatorname{Re}\left(b_{2}\right)=0$. Let $a=a_{2} / 2 i$ and $b=b_{2} / 2 i$; then $a \neq 0$ and $b$ are real constants. Again from (3), we get

$$
f(z)=\frac{1}{K-1}\left(e^{2 i(a z+b)}-1\right), \quad g(z)=\frac{K}{1-K}\left(e^{-2 i(a z+b)}-1\right) .
$$

Thus

$$
f(z)-1=\frac{1}{K-1}\left(e^{2 i(a z+b)}-K\right) .
$$

Let $K_{1}+i K_{2}=\frac{1}{2} \log K$, where $K_{1}$ and $K_{2}$ are real constants. From (5), we obtain $z_{0}=\left(K_{2}-b\right) / a-i K_{1} / a$ is a one-point of $f$. Since $z_{0}$ is real, we obtain $K_{1}=0$. Let $c=b-K_{2}$; then $c$ is a real constant. Again from (4), we have

$$
\begin{aligned}
& f(z)=\frac{e^{2 i(a z+b)}-1}{e^{2 i(b-c)}-1}=\frac{\sin (a z+b)}{\sin (b-c)} e^{i(a z+c)}, \\
& g(z)=\frac{e^{2 i(b-c)}}{1-e^{2 i(b-c)}}\left(e^{-2 i(a z+b)}-1\right)=\frac{\sin (a z+b)}{\sin (b-c)} e^{-i(a z+c)} .
\end{aligned}
$$

Assume that $a_{3} \neq 0$. Applying Lemma 3 to (2), we have

$$
a_{2} z+b_{2}=p\left(a_{3} z+b_{3}\right)+2 q \pi i,
$$


where $p \neq 0,1$ and $q$ are integers. Thus

$$
f(z)=\frac{e^{p\left(a_{3} z+b_{3}\right)}-1}{e^{\left(a_{3} z+b_{3}\right)}-1}, \quad g(z)=\frac{e^{-p\left(a_{3} z+b_{3}\right)}-1}{e^{-\left(a_{3} z+b_{3}\right)}-1} .
$$

If $p \neq-1$, then $z_{3}=2 m \pi i / p a_{3}-b_{3} / a_{3}(m$ is an integer $\neq 0, \bmod (p))$ and $z_{4}=$ $z_{3}+2 \pi i / a_{3}$ are two zeros of $f$. If $p=-1$, then $z_{5}=\pi i / a_{3}-b_{3} / a_{3}$ and $z_{6}=$ $z_{5}+2 \pi i / a_{3}$ are two one-points of $f$. Since $z_{3}, z_{4}, z_{5}$, and $z_{6}$ are real, we obtain $\operatorname{Re}\left(a_{3}\right)=\operatorname{Re}\left(b_{3}\right)=0$. Let $a=a_{3} / 2 i$ and $b=b_{3} / 2 i$; then $a \neq 0$ and $b$ are real constants. Again from (6), we get

$$
\begin{aligned}
& f(z)=\frac{e^{2 p i(a z+b)}-1}{e^{2 i(a z+b)}-1}=\frac{\sin (p(a z+b))}{\sin (a z+b)} e^{i(p-1)(a z+b)}, \\
& g(z)=\frac{e^{-2 p i(a z+b)}-1}{e^{-2 i(a z+b)}-1}=\frac{\sin (p(a z+b))}{\sin (a z+b)} e^{-i(p-1)(a z+b)} .
\end{aligned}
$$

This completes the proof of Theorem 2 .

\section{Proof of Theorem 1}

We note that in [1] in fact Czubiak and Gundersen proved the following results:

Theorem C. If $f$ and $g$ are in $\mathcal{K}$ and share 0 and 1 IM and they are also both real on the real axis, then $f$ and $g$ share 0 and $1 C M$.

Theorem D. If $f$ and $g$ are in $\mathcal{K}$ and share 0 and 1 IM and $f$ is real for real $z$ and $g$ is not real for some real value of $z$, then $f$ and $g$ must satisfy exactly one of the following relations:

$$
f(z)=\sin ^{2}(a z+b) \quad \text { and } \quad g(z)=-i \sin (a z+b) e^{i(a z+b)},
$$

where $a \neq 0$ and $b$ are real constants;

$$
f(z)=\frac{\sin ^{2}(p(a z+b))}{\sin ^{2}(a z+b)} \quad \text { and } \quad g(z)=\frac{\sin (p(a z+b))}{\sin (a z+b)} e^{i(p-1)(a z+b)},
$$

for $p=-2$ and for $p=-3$, where $a \neq 0, b$ are real constants;

$$
\begin{aligned}
& f(z)=1-\frac{(g(z)-1)^{2}}{e^{2 p i(a z+b)}}=1-\frac{\sin ^{2}((p-1)(a z+b))}{\sin ^{2}(a z+b)}, \\
& g(z)=\frac{\sin (p(a z+b))}{\sin (a z+b)} e^{i(p-1)(a z+b)},
\end{aligned}
$$

for $p=3$ and for $p=4$, where $a \neq 0, b$ are real constants.

Using Lemma 2, we have the following result:

Theorem 3. If $f$ and $g$ are in $\mathcal{K}$ and share 0 and 1 IM and neither $f$ nor $g$ is real for all real $z$, then $f$ and $g$ share 0 and $1 C M$.

Proof. Since $f$ and $g$ are in $\mathcal{K}$ and neither $f$ nor $g$ is real for all real $z, f$ and $g$ must have the forms in Lemma 2. Therefore, $f$ and $g$ have only simple zeros. To see that $f$ and $g$ have only simple one-points, one can consider $F=1-f$ and $G=1-g$ and use the same reasoning. Theorem 3 follows.

It is obvious that the functions $f(z)$ and $g(z)$ in the relations (ii) and (iii) of Theorem 1 are not real for all real $z$. From Theorem 2, Theorem C, and Theorem 3 , we have the following results: 
Theorem $\mathbf{C}^{\prime}$. If $f$ and $g$ are in $\mathcal{K}$ and share 0 and 1 IM and they are also both real on the real axis, then $f \equiv g$.

Theorem $3^{\prime}$. If $f$ and $g$ are in $\mathcal{K}$ and share 0 and 1 IM and neither $f$ nor $g$ is real for all real $z$, then $f$ and $g$ must satisfy exactly one of the relations (i), (ii), and (iii) of Theorem 1.

Now using Theorem $\mathrm{C}^{\prime}$, Theorem $3^{\prime}$, and Theorem $\mathrm{D}$, we finally obtain the conclusion of Theorem 1.

\section{Correction of a Result of Osgood And Yang}

In [1], Czubiak and Gundersen used the following result obtained by C. F. Osgood and C. C. Yang:

Theorem $\mathbf{E}$ ([3, Theorem 3]). If $f$ and $g$ are nonconstant entire functions of finite order and share 0 and $1 C M$, then either $f \equiv g$ or

$$
f(z)=1+e^{Q}+\cdots+e^{(n-1) Q}=\left(1-e^{n Q}\right) /\left(1-e^{Q}\right)
$$

and

$$
g(z)=1+e^{-Q}+\cdots+e^{-(n-1) Q}=\left(1-e^{-n Q}\right) /\left(1-e^{-Q}\right),
$$

or more generally

$$
f(z)=\left(1-e^{2 \pi i P(Q)}\right) /\left(1-e^{2 \pi i Q}\right)
$$

and

$$
g(z)=\left(1-e^{2 \pi i P(Q)}\right) /\left(e^{2 \pi i(P(Q)-Q)}-e^{2 \pi i P(Q)}\right),
$$

where $Q$ is a polynomial in $z$ and $P(z)$ is a polynomial in $z$ with rational numbers as the coefficients.

The above result of Osgood and Yang, however, is not true for

$$
f(z)=\left(\frac{1}{2}+\mathrm{id}\right)\left(1-e^{i z}\right) \text { and } g(z)=\left(\frac{1}{2}-\mathrm{id}\right)\left(1-e^{-i z}\right)
$$

where $d$ is a real constant. The following result is a correction of Theorem E:

Theorem $\mathbf{E}^{\prime}$. If $f$ and $g$ are nonconstant entire functions of finite order and share 0 and $1 C M$, then $f$ and $g$ must satisfy exactly one of the following relations:

$$
\begin{aligned}
& f \equiv g ; \\
& f(z)=\frac{1}{K-1}\left(e^{P(z)}-1\right) \quad \text { and } g(z)=\frac{K}{1-K}\left(e^{-P(z)}-1\right),
\end{aligned}
$$

where $K \neq 0,1$ is a constant, and $P(z)$ is a nonconstant polynomial;

$$
\begin{aligned}
& f(z)=\left(1-e^{n Q(z)}\right) /\left(1-e^{Q(z)}\right), \\
& g(z)=\left(1-e^{-n Q(z)}\right) /\left(1-e^{-Q(z)}\right),
\end{aligned}
$$

where $n \neq 0,1$ is an integer, and $Q(z)$ is a nonconstant polynomial;

$$
\begin{aligned}
& f(z)=\left(1-e^{2 \pi i P(Q(z))}\right) /\left(1-e^{2 \pi i Q(z)}\right), \\
& g(z)=\left(1-e^{-2 \pi i P(Q(z))}\right) /\left(1-e^{-2 \pi i Q(z)}\right),
\end{aligned}
$$

where $Q(z)$ is a nonconstant polynomial in $z$, and $P(z)$ is a polynomial in $z$ with rational numbers as the coefficients, and $\operatorname{deg}(P) \geq 2$. 
We may rewrite the functions $f(z)$ and $g(z)$ in the relation (iii) of Theorem $\mathrm{E}^{\prime}$ in the following forms:

$$
\begin{aligned}
& f(z)=1+e^{Q(z)}+\cdots+e^{(n-1) Q(z)} \\
& g(z)=1+e^{-Q(z)}+\cdots+e^{-(n-1) Q(z)}
\end{aligned}
$$

where $n \geq 2$;

(iii) $)_{\mathrm{b}}$

$$
\begin{aligned}
& f(z)=-e^{-m Q(z)}-e^{-(m-1) Q(z)}-\cdots-e^{-Q(z)}, \\
& g(z)=-e^{m Q(z)}-e^{(m-1) Q(z)}-\cdots-e^{Q(z)},
\end{aligned}
$$

where $m=-n \geq 1$.

Next we proceed to prove Theorem $\mathrm{E}^{\prime}$.

By the assumption of Theorem $\mathrm{E}^{\prime}$, we have two polynomials, $R(z)$ and $S(z)$, such that

$$
f=g e^{R} \quad \text { and } \quad f-1=(g-1) e^{S} .
$$

Suppose that $f \not \equiv g$. Then $e^{S} \not \equiv 1$ and $e^{S-R} \not \equiv 1$. Thus from (7) we obtain

$$
\begin{gathered}
f(z)=\left(1-e^{S(z)}\right) /\left(1-e^{S(z)-R(z)}\right), \\
g(z)=\left(1-e^{-S(z)}\right) /\left(1-e^{R(z)-S(z)}\right) .
\end{gathered}
$$

We discuss the following two cases.

(A) Assume that $e^{S(z)-R(z)} \equiv K$, where $K$ is a constant. Then $K \neq 0,1$. Thus from (8) we have

$$
f(z)=\frac{1}{K-1}\left(e^{P(z)}-1\right) \quad \text { and } \quad g(z)=\frac{K}{1-K}\left(e^{-P(z)}-1\right),
$$

where $P(z)=S(z)$ is a nonconstant polynomial.

(B) Assume that $e^{S(z)-R(z)}$ is not a constant. In [3], in fact Osgood and Yang proved that in this case $f(z)$ and $g(z)$ must satisfy exactly one of the relations (iii) and (iv) of Theorem $\mathrm{E}^{\prime}$. Theorem $\mathrm{E}^{\prime}$ is thus proved.

\section{ACKNOWLEDGMENT}

I am grateful to the referee for his valuable comments.

\section{REFERENCES}

1. T. P. Czubiak and G. G. Gundersen, Entire functions that share real zeros and real ones, Proc. Amer. Math. Soc. 82 (1981), 393-397. MR 82h:30028

2. A. Edrei, Meromorphic functions with three radially distributed values, Trans. Amer. Math. Soc. 78 (1955), 276-293. MR 16:808d

3. C. F. Osgood and C. C. Yang, On the quotient of two integral functions, J. Math. Anal. Appl. 54 (1976), 408-418. MR 53:8421

Department of Mathematics, Shandong University, Jinan, Shandong, 250100, People's Republic of China 